\title{
Electroencephalogram Signals Processing for the Diagnosis of Petit mal and Grand mal Epilepsies Using an Artificial Neural Network
}

\author{
M. R. Arab* ${ }^{1}$, A. A. Suratgar ${ }^{2}$, V. M. Martínez-Hernández ${ }^{2}$, A. Rezaei Ashtiani ${ }^{3}$ \\ ${ }^{1}$ Department of Biomedical Engineering, Arak Medical University, Arak, Iran. \\ *a-suratgar@aut.ac.ir \\ ${ }^{2}$ Department of Electrical Engineering, Arak University, Arak, Iran. \\ ${ }^{2}$ Department of Electrical Engineering, Amirkabir University of Technology(Tehran Polytechnic), Tehran, Iran. \\ ${ }^{3}$ Department of Neurology, Arak Medical University, Arak, Iran.
}

\begin{abstract}
In this study, a novel wavelet transform-neural network method is presented. The presented method is used for the classification of grand mal (clonic stage) and petit mal (absence) epilepsies into healthy, ictal and interictal (EEGs). Preprocessing is included to remove an artifact occurred by blinking and a wandering baseline (electrodes movement) as well as an eyeball movement artifact using the Discrete Wavelet Transformation (DWT). Denoising EEG signals from the AC power supply frequency with a suitable notch filter is another job of preprocessing. The preprocessing enhanced speed and accuracy of the processing stage (wavelet transform and neural network). The EEGs signals are categorized into normal and petit mal and clonic epilepsy by an expert neurologist. The categorization is confirmed by the Fast Fourier Transform (FFT) analysis. The dataset includes waves such as sharp, spike and spike-slow wave. Through the Countinous Wavelet Transform (CWT) of EEG records, transient features are accurately captured and separated and used as classifier input. We introduce a two-stage classifier based on the Learning Vector Quantization (LVQ) neural network localized in both time and frequency contexts. The particular coefficients of the Continuous Wavelet Transform (CWT) are networks. The simulation results are very promising and the accuracy of the proposed method obtained is of about $80 \%$.
\end{abstract}

Keywords: Tonic-clonic epilepsy, petit mal epilepsy, Continuous Wavelet Transform (CWT), absence epilepsy

\section{RESUMEN}

En este estudio, se presenta un nuevo método basado en redes neuronales y la transformada ondicular. El método presentado se usa para la clasificación de la epilepsia gran mal (clónica) y pequeño mal (de ausencia) en saludable, ictal e interictal (EEG). Se incluye el pre procesamiento para eliminar un artefacto causado por el parpadeo y una línea de base errante (movimiento de electrodos) así como un artefacto producido por el movimiento ocular usando la Transformada Ondicular Discreta (DWT). Otra función del pre procesamiento es la eliminación de ruido de las señales de EEG de la frecuencia de la fuente de alimentación AC con un filtro de eliminación adecuado. El pre procesamiento aumentó la velocidad y precisión de la etapa de procesamiento (transformada ondicular y red neuronal). Un neurólogo experto clasifica las señales de EEG en epilepsia normal, pequeño mal y clónica. La clasificación se corrobora por medio del análisis con Transformada Rápida de Fourier (FFT). El conjunto de datos incluye ondas tales como agudas, puntas y punta-onda lenta. Mediante la Transformada Ondicular Continua (CWT) de los registros del EEG, se capturan y separan correctamente características transitorias y se usan como entrada del clasificador. Introducimos un clasificador de dos etapas basado en redes de cuantización vectorial (LVQ) localizado en los contextos tiempo y frecuencia. Los coeficientes particulares de la Transformada Ondicular Continua (CWT) son redes. Los resultados de la simulación son muy prometedores y la exactitud del método propuesto es de alrededor del $80 \%$.

\section{Introduction}

Epilepsy is a common neurological condition that affects millions of people throughout the world. The cause of this condition is a disorder in the electrical activity of the brain cortex. The disease

epilepsy is characterized by a sudden and recurrent malfunction of the brain that is termed "seizure". Epileptic seizures reflect the clinical signs of an excessive and hyper synchronous activity of neurons in the brain. The electroencephalogram 
(EEG), a high complex signal is one of the most common sources of information used to study brain function and neurological disorders. Among the non-invasive techniques for probing human brain dynamics, the electroencephalogram (EEG) provides a direct measure of cortical activities with millisecond and temporal resolution. The EEG is the record of the electrical potential generated by the cerebral cortex nerves cell analysis.

Neural network detection systems have been proposed by some researches. Pradhan et al ${ }^{[16]}$ used raw EEG as input of neural networks. Weng and Khorasani ${ }^{[8]}$ used the feature proposed by Gotman with an adaptive neural network but his result shows a poor false detection rate ${ }^{[13]}$.

Nonlinear analyses like wavelet transform and neural networks should be performed in the case of EEG signals. Petrosian et al. ${ }^{[7]}$ showed the ability of specifically designed and trained recurrent neural networks combined with wavelet preprocessing to predicate onset of epileptic seizures both on scalp and intracranial recording only one channel of EEG. Subasis et al. ${ }^{[4]}$ classified EEG signals using neural networks and logistic regression. Adeli et al. ${ }^{[3]}$ classified EEG signals using mixed band wavelet-chase-neural network.

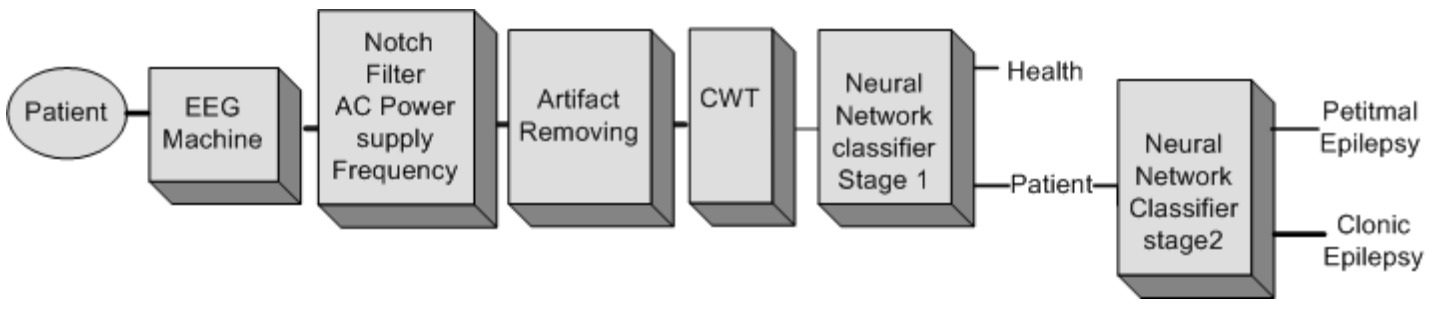

Figure 1. Block diagram methodology for diagnosing epilepsy.
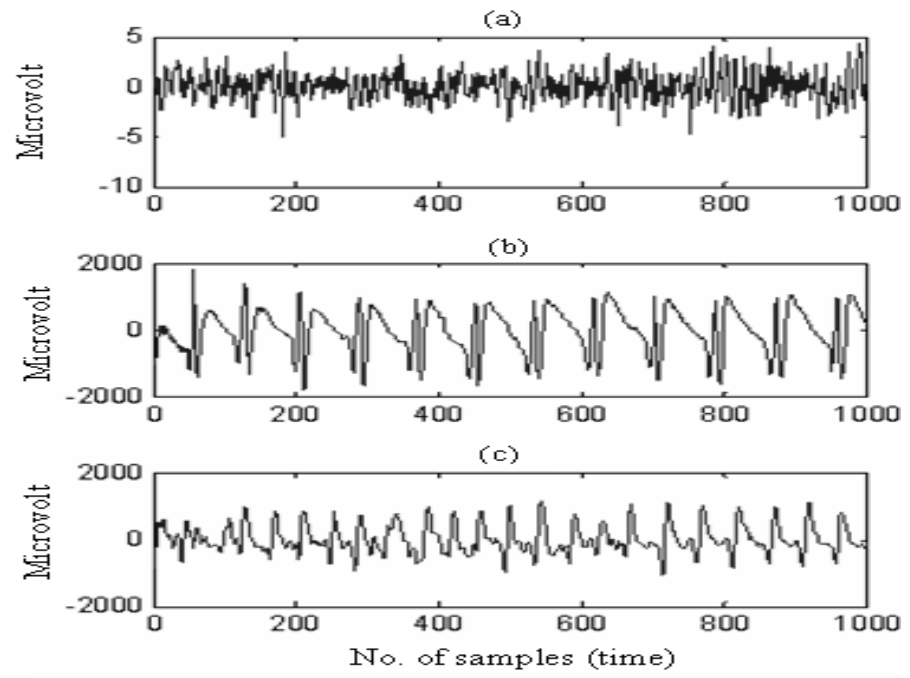

Figure 2. EEG signals. (a) Normal, (b) Absence and (c) Grand mal. 


\section{Materials and method}

Epilepsy: Epileptic seizures are the result of the transit and unexpected electrical disturbance of the brain. About one percent of people will experience a seizure at some time in their lives.

The excitability of the Central Nervous System (CNS) causes abnormality in the electrical activities and in EEG an abnormality pattern is observed as its amplitude dramatically increases. Epilepsy is one of the neurological conditions in which the inhibitory system of nerve cells is involved. On the other hand, excitability of nerve cells increases this way.

Seizures may occur in a part of one hemisphere, generally in the temporal or frontal lobe because of which they are called partial seizures. They are seen in every channel of the EEG signal beginning. Seizures involving the whole brain are called generalized seizures. They are seen in every channel of the EEG signal. Tonic-clonic seizures (formerly called "grand mal") and typical absence (formerly called "petit mal") are related to generalized seizures.

Epileptiform activity refers to the spikes, spikeslow waves and sharp waves. In absence epilepsy, there is typical seizure activity in the form of spikeslow wave with frequency of $3 \mathrm{~Hz}$ shown in Fig. 2 and 3 , but in grand mal epilepsy, this seizure activity generates poly spikes (poly spikes and waves) or poly sharp waves with frequency $4 \mathrm{~Hz}$ [1] as shown in Fig. 2 and 3.

Diagnosis of epilepsy using the Wavelet Transform: A powerful method was proposed in the late 1980s to perform time-scale analysis of signals. The properties of the Wavelet Transform
(WT) representation can be used to analyze various transient events in bio-signals. One area in which the WT has been particularly successful is the epileptic area because it captures transient features and localizes them in both time and frequency context accurately. The Wavelet Transform is an integral transform for which the set of basis functions, known as wavelets, are localized both in time and frequency. Moreover, the wavelet analysis can be constructed from a signal function $\psi(\mathrm{t})$ by means of translation and dilation:

$$
\psi_{a, b}(t)=a^{-\frac{1}{2}} \psi\left(\frac{t-b}{a}\right)
$$

$\psi(\mathrm{t})$ is commonly referred to as the mother function or analyzing wavelet. The Wavelet Transform of function $\mathrm{h}(\mathrm{t})$ is defined as

$$
\mathrm{W}(\mathrm{a}, \mathrm{b})=\int_{-\infty}^{+\infty} \mathrm{h}(\mathrm{t}) \psi *_{\mathrm{a}, \mathrm{b}}(\mathrm{t}) \mathrm{dt}
$$

where, $\psi *(\mathrm{t})$ denotes the complex conjugate of $\psi(\mathrm{t})$.

The Continuous Wavelet Transform transforms a function or signal of one independent variable $t$ into a function of two independent variables a and b. This approach is redundant and not efficient for algorithm implementations.

Therefore, in practice, the Continuous Wavelet Transform, Eq. 2, can be discretized (DWT) by setting the dilation and translation factors as $a=2$ and $b=1^{[17] \text {. }}$ 
(a)

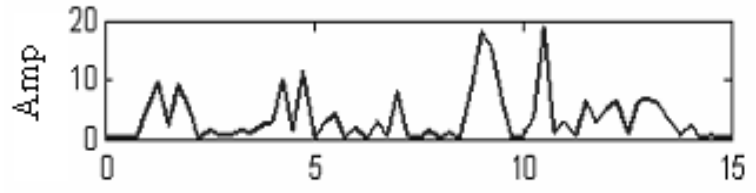

(b)

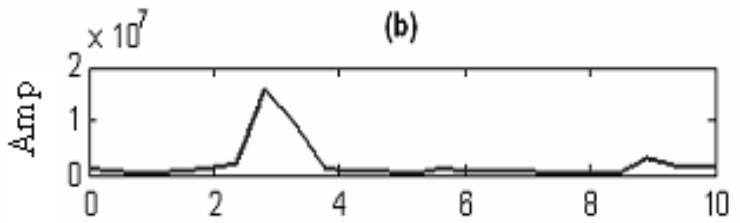

$\times 10^{6}$

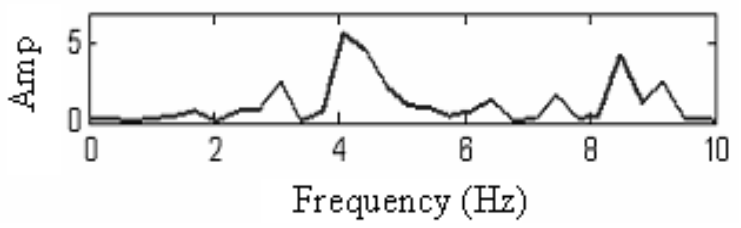

Figure 3. Comparison of power spectral EEG signals: (a) Healthy (b) Absence and

(c) Clonic epilepsy

Common choices for discrete wavelet parameter, a $=2$ and $b=1$, this power-of-two logarithmic scaling of both dilation and translation steps is known as the dyadic grid arrangement. The dyadic grid is perhaps the simplest and most efficient discretization for practical purposes and lends itself to the construction of an orthonormal wavelet basis.

$$
\psi_{\mathrm{mn}}(\mathrm{t})=2^{\frac{\mathrm{m}}{2}} \psi\left(2^{\mathrm{m}} \mathrm{t}-\mathrm{n}\right)
$$

Coefficient Details (D) and Coefficient Approximation (A) are obtained by means of the discrete wavelet transform algorithm.

The hundred EEG segments are used including the spike, sharp and slow wave, artifact and background normal EEG. An artifact is caused by movement of the eyeball and blinking and can corrupt EEG data. Detection and removal of artifacts is performed using the Discrete Wavelet Transform (DWT).
In this method, we apply the bior 3.3 wavelet function, since the shape of its mother wavelet resembles the shape artifact in Fig. $5 a$.

Subjects and data recording and processing: The EEG data used in this study with segments of 1024 samples (5.91 second duration) were sampled at $173 \mathrm{~Hz}^{[10]}$. The performance of the classifier is assessed by selecting 100 signals containing epileptiform events: spike or sharp wave, spikeslow wave complex, EMG artifact and background normal EEG. Electrod locations and names are specified by the International $10-20$ system. We used Matlab software version 7.2 for simulation and data processing. The EEG datasets belonging to three subject groups is as following:
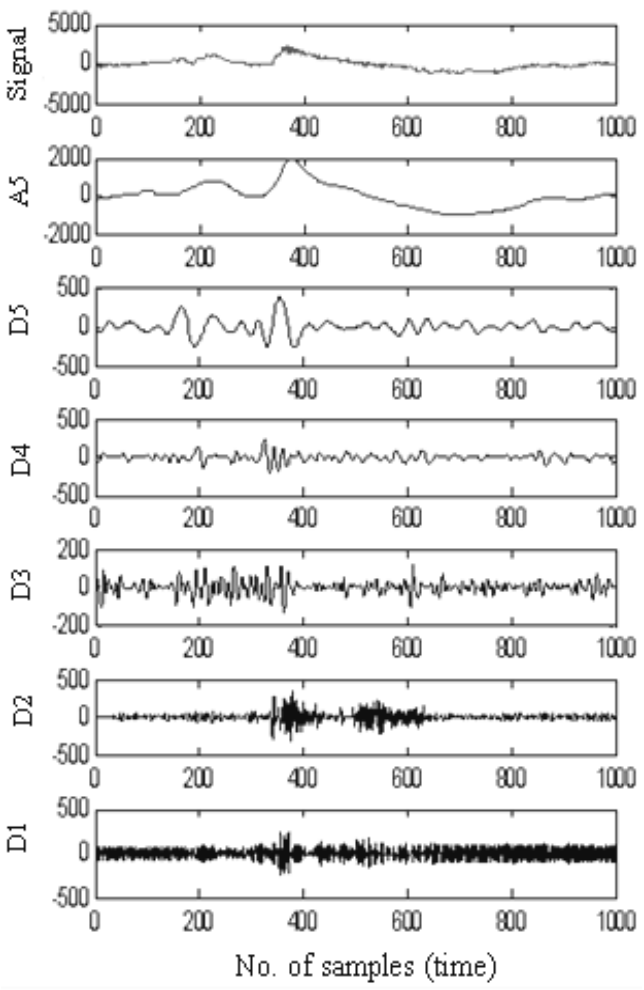

Figure 4. Artifact removal using decomposition signal at level 5 . 
- Healthy subjects (normal EEG) were recorded in open and closed eye condition

-Epileptic subjects during a seizure-free interval (interictal EEG)

-Epileptic subjects during a seizure (ictal EEG)

Artifact removal: contamination of EEG data can occur at many points during the recording process. Most of the artifacts considered here are caused by movements of the eyeball and blinking and can corrupt EEG data. They produce a high amplitude signal that many times can be greater than the EEG signals of interest. Because of its high amplitude, an eye blink can corrupt data on all electrodes. This artifact's diffusion across the scalp is stronger than that of the eye blink artifact. Eye blinks and movements often occur at close intervals producing an effect shown in Fig. 4. For detection and removal of the artifact, the Discrete Wavelet Transform (DWT) was used.

In this method, we applied the bi-orthogonal wavelet (Bior3.3) due to its similarity with the artifact. Bior3.3 has linearity phase in wavelet filters. In Fig. 5a, the artifact is compared with wavelet Bior3.3 and the similarity is investigated. The signal of the artifact is decomposed into tree form into two categories in six levels. In decomposition, level five approximations (A5) include artifacts with band frequency $0-2.1 \mathrm{~Hz}$ (lowest band) and there is no subject's epileptic seizure (Fig. 5). Approximation A5 is eliminated and the rest (D1-D5) of coefficients are added, reconstruction gives again the denoising signal (Fig. 6). The signal-to-noise ratio of this method is 22.11.

One of the problems of EEG signals is the existence of noise from AC power supplies $(50 \mathrm{~Hz})$. They are denoised by suitable notch filters. Coefficients a1, a2 are obtained by bilinear method.

$$
\mathrm{H}(\mathrm{z})=\frac{1}{2} \frac{\left(1+\mathrm{a}_{2}\right)-2 \mathrm{a}_{1} \mathrm{z}^{-1}+\left(1+\mathrm{a}_{2}\right) \mathrm{z}^{-2}}{1-\mathrm{a}_{1} \mathrm{z}^{-1}+\mathrm{a}_{2} \mathrm{z}^{-2}}
$$

$a 1=-1.5162 \quad a 2=0.9615$

Feature extraction by CWT: Feature Extraction information signals using the Continuous Wavelet Transform (CWT) with a set of resolution scales. According to Fig. 5b, the wavelet function (Mexican-hat) with spike is similar. The Mexicanhat equation is according to Eq. 5 :

$$
\psi(x)=\left(\frac{2}{\sqrt{3}} \pi^{\frac{-1}{4}}\right)\left(1-x^{2}\right) e^{-x^{2} / 2}
$$

The first step in the analysis is the normalization of the wavelet coefficients to reduce the dependence on the amplitude of the EEG signals.

Whole scales 3-37 CWT cover all information of epilepsies shown in Fig. 7. In Fig. 7, we can see energy of signals associated to slow waves.

An overview of neural networks: In this study we employ the Learning Vector Quantization (LVQ) neural network for design classification. The architecture of LVQ consists of two layers. (a)

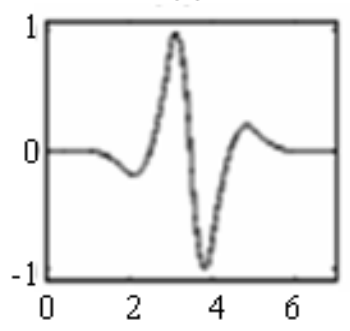

(b)

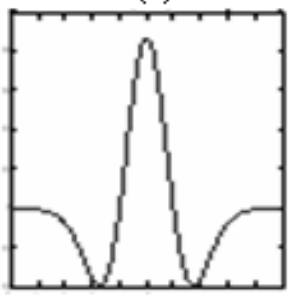

Figure 5. Wavelet functions. (a) Bior3.3 (b) Mexican hat.

First competitive layer and a second linear layer.: The competitive layer learns to classify input vectors in much the same way as the competitive layers transform the competitive layer's class into target Classifications defined by the user: We refer to the classes learned by the competitive layer as subclasses and the classes of the linear layer as target classes. The output of the neural network has two models (yes/no or $1 / 2$ ) according to what is shown in Fig. $8^{[15,24] \text {. }}$ 
With this software, the accuracy of the diagnosis of petit mal and grand mal epilepsies is of about $80 \%$.
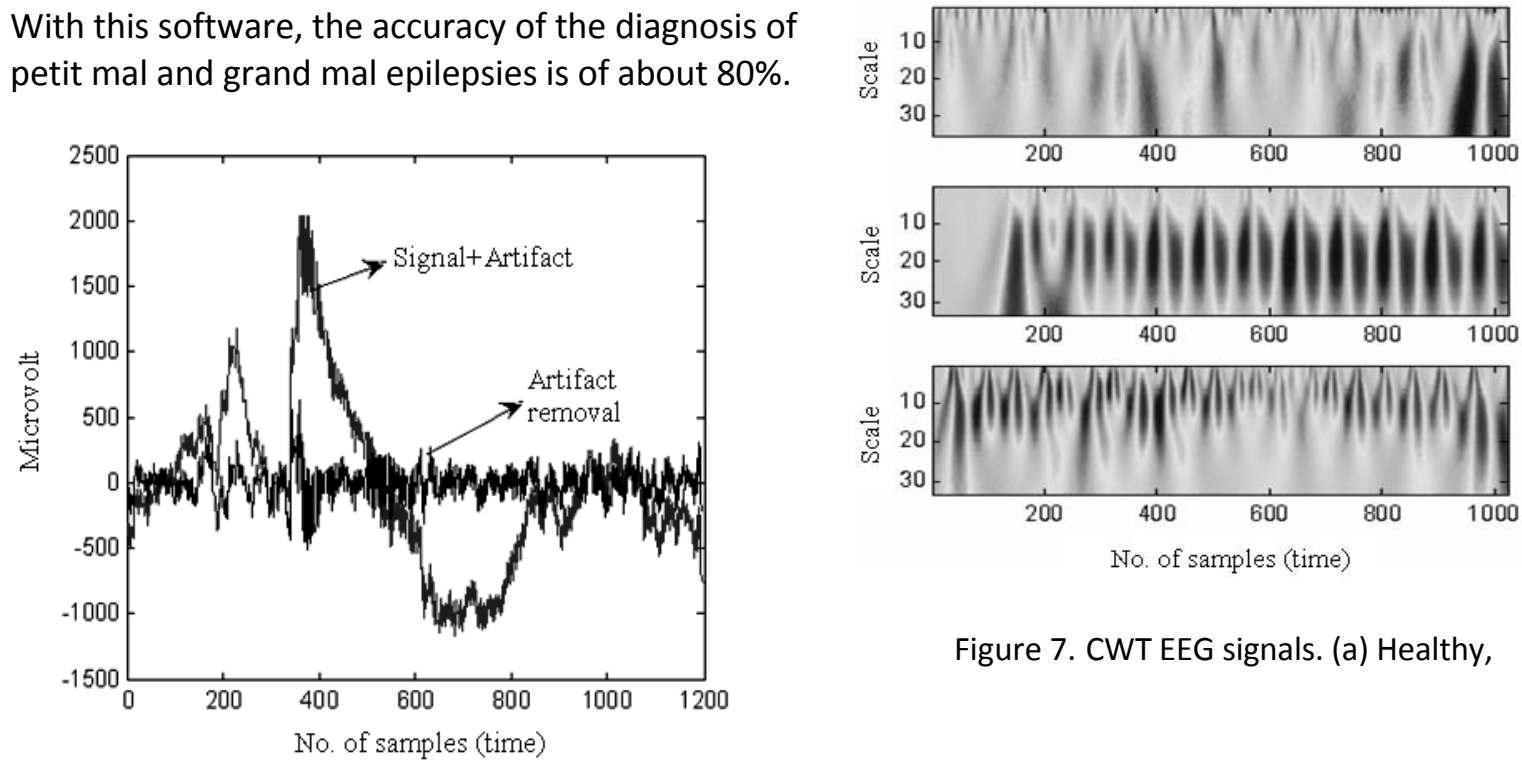

Figure 7. CWT EEG signals. (a) Healthy,

Figure 6. Artifact removal using DWT

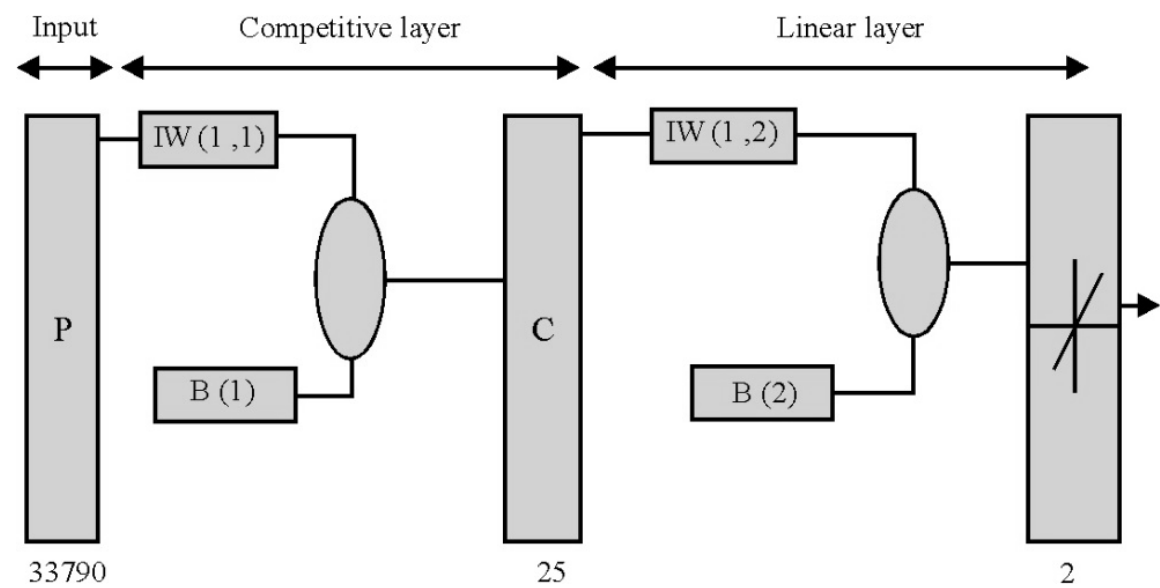

Figure 8. Architecter LVQ neural network (b) Petit mal seizures and (c) Clonic seizuresMexican hat. 
Table 1: Results of simulation

\begin{tabular}{|c|c|c|c|c|}
\hline EEG Signals & $\begin{array}{l}\text { No. } \\
\text { signals } \\
\text { input NN }\end{array}$ & $\begin{array}{l}\text { No. signals } \\
\text { diagnosis } \\
\text { NN }\end{array}$ & $\begin{array}{l}\text { Error } \\
\mathrm{NN}\end{array}$ & Accuracy \\
\hline Healthy & 35 & 28 & $20.00 \%$ & $80.00 \%$ \\
\hline Petit mal Epilepsy & 35 & 30 & $14.20 \%$ & $85.71 \%$ \\
\hline Clonic Epilepsy & 30 & 23 & $23.30 \%$ & $76.66 \%$ \\
\hline
\end{tabular}

\section{Results}

In this study, the accuracy of the diagnosis of petit mal and grand mal epilepsies achieved is of about $80 \%$.

We used actual measured EEG signals for learning the neural network. We used one part of the data for learning and other part for testing. Table 1 shows the result of the neural network for the classification of healthy, petit mal and clonic signals and recognition of two kinds of epilepsy.

The segments of epileptic signals are separated by the neurologist and analyzed by means of the Fast Fourier Transform (FFT) that can show the sign of epileptic signals well, which can confirm the result of the neural network.

\section{Discussion}

The advantages of this method: The proposed method introduced an intelligent diagnosis for epilepsy (petit mal and clonic) and, also, automatically detected healthy and epileptic patients. In previous research, only automatic detection of healthy and epileptic patients has been done.

The performance of preprocessing and selection of coefficients of the Continuous Wavelet Transform (CWT) precisely enables the neural network to increase the ability of diagnosis.

The epilepsy classification (petit mal and clonic) using EEG is one of the heavy duties of neurologists $[1,2,11,18]$. One of the other advantages of the proposed method is to help neurologists to detect and classify sickness clearly.

Noise and artifacts cancelling is a useful character of the proposed method.

The accuracy of the proposed method is shown in Table 1.

The limitation of this method: The proposed method can be used to classify two kinds of manifestations of epilepsy. At present, the authors are working on the detection of more kinds of epilepsies and the results will be presented in future.

The computation of the proposed method is time consuming. In order to achieve real-time computation, an advanced processor is advised.

\section{Conclusion}

In this study, a novel wavelet transform-neural network method was presented. The presented method was used for the classification of epilepsies of grand mal (clonic stage) and petit mal (absence) type into healthy, ictal and interictal (EEGs). The preprocessing enhanced speed and accuracy of the processing stage (wavelet transform and neural network).

The EEGs signals were categorized into normal, petit mal and clonic epilepsy by an expert neurologist. The categorization was confirmed by the Fast Fourier Transform (FFT) analysis.

The simulation results are very promising and the accuracy of the proposed method obtained is of about $80 \%$. 


\section{References}

[1] W. G. Bradley, W. H. Trescher and R. P. Lesser, Neurology in Clinical Practice. 3rd Ed., 2004, pp: 710762.

[2] F. H. Duffy, V. G. Iyer and W. W. Surwill, Clinical Electroencephalography and Topographic Brain Mapping. Springer Verlag, New York, 1989, pp: 210270.

[3] H. Adeli, N. Dadmeher and S. Ghosh-Dastidar, 2007. Mixed-band wavelet-chaos-neural network methodology for epilepsy and epileptic seizure detection. IEEE Trans. Biomed. Eng., Vol:54, 2007, pp: 545-1551.

[4] A. Subasi, E. Ercelebi, Classification of EEG signals using neural network and logistic regression. Comput. Methods Program. Biomed., Vol:78, 2005, pp: 87-99.

[5] T. Zikov, S. Bibian, G. A. Dumont and M. Huzmezan, A Wavelet based de-noising, technique for occular artifact correction of electroencephalogram, 2007, pp:101-106.

[6] M. Latka, Z. Was, A. Kozik and B. J. West, Wavelet analysis of epileptic spikes. Phys. Rev. E Stat. Nonlin Soft Matter Phys., Vol: 67, 2003, pp: 495-500.

[7] A. Petrosian, D. Prokhorov, R. Homan, R. Dascheiff and D. Wunsch, Recurrent neural network based prediction of epileptic seizures in intercranial and extracranial EEG. Neurocomputing, Vol:30, 2000, pp:201-218.

[8] W. Weng, K. Khorasani, An adaptive structure neural network with application to EEG automatic seizure detection. Neural Network, Vol:9, 1996, pp:12231240.

[9] H. R. Mohseni, A. Maghsoudi and M. Shamsollahi, Seizure detection EEG signals: A comparison of different approaches. Proceeding of the IEEE Annual International Conference on Engineering in Medicine and Biology Society, Aug. 30-Sep 3, 2006, pp: 67246727.
[10] R. G. Andrzejak G. Widman, K. Lehnertz, C. Rieke, P. David and C. E. Elger, Epileptic process as nonlinear determinstics dynamics in astochastic environment: An evaluation on mesial temporal lobe epilepsy. Epilepsy Res., Vol: 44, 2001, pp:129-140.

[11] E.Niedermeyer, Silva FLDa.

Electroencephalography. 5th Ed., Lippincott Williams and Wilkings, 2005, pp: 790-800.

[12] D. Novak, L. Lhotska, EEG and VEP signal processing. Cybernetics, Faculty of Electrical Eng, 2004, pp: 50-53.

[13] Y. U. Khan, J. Gotman, 2003. Wavelet based automatic seizure detection in intercerebral electroence phalogram . Clin. Neurophsiol., Vol:114, 2003, pp: 898-908.

[14] S. Mallat, A Wavelet Tour of Signal Processing. 2nd Edn., Academic Press, San Diego, 1999, pp: 637-653.

[15] M. B. Menhaj, Fundamentals of neural network. Amir Kabir press, 2005, pp:700-730.

[16] N. Pradhan, P. K. Sadasivan and G. R. Arunodaya, Detection of seizure activity in EEG by an artificial neural network. A preliminary study. Comput. Biomed., Res. Vol:29, 1996, pp:303-313.

[17] P. Addison, Illustrated Wavelet Transform Handbook. 1st Edn., Taylor and Francis, USA., ISBN: 10: 0750306920, 2002, pp: 7-17.

[18] M. Aminoff, Electroencephalography: General Principles and Clinical Application Applications. In: Electrodiagnosis in Clinica Neurology. Library of Congress Cataloging in Publication Data, 2005, pp: 3780.

[19] M. E. Ohadi, Electroencephalography. Etelat, 1998, pp: 700-790.

[20] D. Willam, The Nervous System. In: Physiology, Berne, R.M., M.N. Levy, B.M. Koeppen and B.A. Stanton (Eds.). New York, 2004, pp: 62-206. 
[21] I Rampil , A primer for EEG signal processing in anesthesia. Anesthesiology, Vol:89, 1998,pp: 9801002.

[23] A. Mertins, Signal Analysis Wavelet Filter Banks. Time-Frequency Transform and Application., Mc Graw Hill, 2002.

[24] The Mathwork Inc. MA Matlab Userss Guide. Wavelet and Nntool Toolbox. 2007.

[25] L. D. lasemidis, D. S. Shiar, C. Wongse, J. C. Sackelles, P. M. Pradalos and J. C. Principe, Adaptive epileptic seizure predication system. IEEE Trans. Biomed. Eng., Vol:50, 2003, pp: 616-627. 


\section{Authors' Biography}

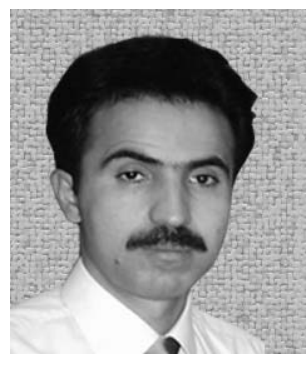

\section{R. Arab}

He received his A.S. degree in nursing from Hamedan Medical University in 1989 and the B.S. and M. S. degrees in electronic from Arak Azad University, Arak, Iran, in 1996 and 2005, respectively. Currently he is with the Biomedical Engineering Department at Arak Medical University. The areas of his research interest are properties of EEG signals including discovering the conjunction between GCS (Glasco Coma Scale) of patients and Bispectral (BIS), presenting a pattern to investigate the level of consciousness of patient, brain mapping and epilepsy. He has published 4 papers in conferences and journals.

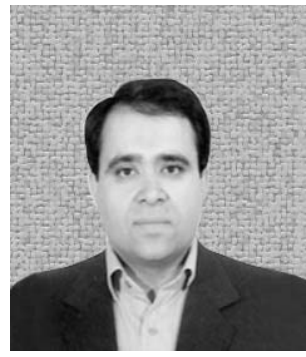

\section{Amir ABOLFAZL- SURATGAR}

He was born in Arak, Iran, in 1974. He received his B. S. degree in electrical engineering from Isfahan University of Technology, Isfahan, Iran, in 1996 with honors and the M. S. and Ph. D. degrees in control engineering from Amirkabir University of Technology (Tehran polytechnic), Tehran, Iran, in 1999 and 2002, respectively. He is assistant professor of Electrical Engineering at the University of Arak, Arak, Iran. Dr. Suratgar received the Outstanding Research Award from the Faculty of Engineering of University of Arak in 2006 and 2005. He received the Outstanding Education Award from the Faculty of Engineering of University of Arak in 2009. Currently, he is with Amirkabir University of Technology (Tehran polytechnic), Tehran, Iran. His research interests are system stability, system modeling and micro electro mechanical systems. He has published more than 50 papers in ccientific Journals and conferences.

\section{A. R. REZAEI-ASHTIANI}

He received his general physician (G. P.) and neurological specialist degrees from Iran University of Medical Science in Tehran, Iran, in 2003 and 2007, respectively. He is currently assistant professor of neurology at Arak University of Medical Science. His research interests focus on epilepsy, multiple sclerosis, EEG and nervous network. He has published more than five journal papers on these areas and twelve papers in conferences. 